\title{
BESTIMMUNG DER KOHÄSIONSENERGIE- UND VERNETZUNGSDICHTE VON POLYMEREN MIT HILFE VON QUELLUNGSMESSUNGEN
}

\author{
Vesela Stoycheva, Harald Goering, Dietmar Wolff, Michael Herzog
}

\section{Zusammenfassung}

Bei der Abschätzung der Beständigkeit von Kunststoffen gegenüber niedermolekularen Flüssigkeiten ist die Kohäsionsenergiedichte CED ein wichtiger Parameter auf molekularer Ebene. Die Vernetzungsdichte $v$ eines polymeren Festkörpers bildet hierbei einen bedeutsamen Kennwert, der darüber hinaus neben der Löslichkeit wichtige Eigenschaften wie thermische Stabilität und Steifigkeit des Materials charakterisiert.

Für die experimentelle Bestimmung von CED und $v$ eignet sich die Untersuchung des Quellverhaltens in unterschiedlichen Lösungsmitteln. Es werden zwei Messmethoden miteinander verglichen und die Ergebnisse unter Berücksichtigung des molekularen Aufbaus von Proben aus Polyurethan (PU) und hochmolekularem - (HMWPE) und ultrahochmolekularem Polyethylen (UHMWPE) diskutiert. Zum Verständnis des Quellverhaltens dieser Polymerproben in niedermolekularen Lösungsmitteln werden neben den Messergebnissen Literaturdaten zu Grunde gelegt.

\section{Abstract}

The Cohesion Energy Density CED is an important parameter on the molecular level for the estimation of the durability of polymers against low molecular solvents. Furthermore the density of cross-linking $v$ in a polymer solid is a significant value for the solubility and other properties like thermal stability and stiffness of the material.

A suitable method for the experimental determination of CED and $v$ is the investigation of the swelling behavior in different solvents. Two measuring methods are compared and the results are discussed under the consideration of the molecular structure of polyurethane (PU), high molecular weight polyethylene (HMWPE) and ultrahigh molecular weight polyethylene (UHMWPE) samples. The theoretical understanding of the swelling behavior of polymers in low molecular solvents is supported by literature values.

\section{EINLEITUNG}

Die Kenntnis der Kohäsionsenergiedichte CED als Quadrat der Löslichkeitsparameter $\delta$ und der Vernetzungsdichte $v$ eines Polymers ist notwendig, um seine mechanischen und Lösungseigenschaften auf molekularer Basis interpretieren zu können. Die experimentelle Bestimmung von $\delta$ und $v$ kann mit Hilfe von Quellungsmessungen erfolgen (Flory 1953, Hanse 2012). Die statistische Theorie hauptvalenzmäßig vernetzter Mischphasen liefert die Verknüpfung der gemessenen thermodynamisch-makroskopischen Größe des Quellungsgrades mit molekularen Parametern. Die Ergebnisse der statistischen Theorie werden allerdings fast immer unter einschränkenden Voraussetzungen und Zugrundelegung einfacher Modelle gewonnen. Orientierende Messungen sollen die methodische Eignung von Quellungsmessungen zur Charakterisierung der durch Gamma-Strahlung verursachten Strukturänderungen von hochmole- kularem und ultrahochmolekularem Polyethylen (HMWPE und UHMWPE) sowie ausgewählten Polyurethanen (PU) überprüfen.

\section{THERMODYNAMIK DER QUELLUNG}

Unter Quellung versteht man die Erscheinung, dass eine makromolekulare Substanz mehr oder weniger große Mengen niedermolekularer Flüssigkeiten oder Dämpfe aufnehmen kann, ohne dass der gequollene Festkörper seinen Zusammenhalt und seine Form verliert und eher elastische als viskose Eigenschaften aufweist. Die elastischen Eigenschaften dieser sog. Gele beruhen im Allgemeinen auf einer dreidimensionalen haupt- oder nebenvalenzmäßigen Verknüpfung der Kettenmoleküle. Hauptvalenzmäßig vernetzte Polymere nehmen bei jeder Temperatur $\mathrm{T}$ nur eine begrenzte Flüssigkeitsmenge auf. Im Grenzfall steht eine Gelmischphase, die aus niedermolekularer Flüssigkeit und makromolekularer vernetzter Substanz zusammengesetzt ist, mit der reinen Flüssigkeit im Gleichgewicht.

Die Theorie der Quellung kann bei der allgemeinen Anwendung gegenwärtig nicht vollständig befriedigen. Die Beziehungen werden unter einer Reihe von stark einschränkenden Annahmen abgeleitet. Vor allem harrt das Problem der Erfassung von Wechselwirkungen, die nicht auf Dispersionskräften beruhen, seiner Lösung. Auch treten besondere Probleme bei Mehrphasensystemen wie PE auf, wenn die Phasenstruktur während des Quellungsexperiments verändert wird. Dennoch werden bei vergleichenden Experimenten die qualitativen Verhältnisse richtig beschrieben. Mit hinreichender Vorsicht können quantitative Untersuchungen auch bei stark polaren Substanzen (PU) zumindest orientierende Werte der untersuchten Größen liefern. 


\section{METHODEN DER}

QUELLUNGSMESSUNG

Zur Bestimmung des Quellungsgrades Q gibt es zwei prinzipielle Wege: die Ermittlung der vom Polymer aufgenommenen Flüssigkeitsmenge und die Volumenänderung des Polymeren beim Quellprozess. Am häufigsten wird die gravimetrische Methode benutzt. Eine Polymerprobe der Masse $\mathrm{m}_{2}$ wird in das betreffende Lösungsmittel gebracht. Nach einer für die Einstellung des Quellgleichgewichts notwendigen Zeit wird die Probe aus dem Lösungsmittel genommen, das außen anhaftende Lösungsmittel sorgfältig entfernt und der Probekörper so schnell wie möglich gewogen (bei leichtflüchtigen Lösungsmitteln in einem abgeschlossenen Gefäß im Sättigungsdampf). Der Quellungsgrad ergibt sich dann aus

(1) $\mathrm{Q}=\left(\rho_{2} \mathrm{~m}_{2}\right) /\left(\rho_{1} \mathrm{~m}_{1}\right)$.

$\rho_{2}$ ist die Polymerdichte, $\rho_{1}$ die des Lösungsmittels. Die vom Polymer aufgenommene Lösungsmittelmasse $\mathrm{m}_{1}$ ergibt sich aus der Gesamtmasse der Probe m:

(2) $\mathrm{m}_{1}=\mathrm{m}-\mathrm{m}_{2}$

Die Quellgeschwindigkeit hängt neben dem Polymer-Lösungsmittelsystem und der Temperatur von den Abmessungen der Probe $a b$, da die Quellung ein diffusionskontrollierter Prozess ist. $\mathrm{Zu}$ beachten ist bei der gravimetrischen Methode, dass die
Proben eine möglichst glatte Oberfläche besitzen, um ein vollständiges Abtrocknen zu erleichtern. Es ist zu untersuchen, ob geringe Bestandteile aus der Polymerprobe herausgelöst werden. Das ist vor allem beim durch energiereiche Strahlung belasteten PE zu beachten, da durch Kettenspaltung während der Bestrahlung kurzkettige und damit leichter lösliche Bestandteile entstehen.

Messmethoden der Quellungsgradbestimmung, die die Änderung der Dimensionen der Probe bei der Quellung als Messgröße benutzen, haben den Vorteil, dass weder die Dichte des Lösungsmittels noch die Polymerdichte bekannt zu sein brauchen. Nimmt man an, dass der Quellungsvorgang isotrop verläuft, kann die Bestimmung der Volumenänderung auf die Messung der Änderung einer Längendimension reduziert werden. Unter der Annahme isotroper Quellung ergibt sich der Quellungsgrad aus der relativen Änderung einer Länge $x$ der Probe um $\Delta \mathrm{x}$ bei der Lösungsmittelaufnahme aus der folgenden Beziehung:

(3) $\mathrm{Q}=3 \Delta \mathrm{x} / \mathrm{x}+3(\Delta \mathrm{x} / \mathrm{x})^{2}+3(\Delta \mathrm{x} / \mathrm{x})^{3}$

Zur Quellungsmessung mit Hilfe der relativen Längenänderung der Probe eignet sich besonders eine mikrooptische Methode. Hierbei werden die Dimensionen einer kleinen (folienartigen) Probe mit einem Mikroskop ausgemessen, jeweils in Kontakt mit dem Lösungsmittel. Diese Methode ist besonders von Vorteil, wenn die maximalen Quellungsgrade bestimmt werden sollen, da infolge der geringen Dicke der Probe der Gleichgewichtsquellungswert im Allgemeinen in wenigen Minuten erreicht wird.

\section{BESTIMMUNG DER KOHÄSIONS- ENERGIEDICHTE}

Die konventionellen Methoden zur Bestimmung der CED niedermolekularer Substanzen, wie die Messung der Verdampfungswärme, der Oberflächenspannung oder des Siedepunktes, können bei Polymeren nicht angewendet werden. Deshalb müssen vergleichende Untersuchungen von Löslichkeitseigenschaften und verwandten Phänomenen in einer Reihe von Flüssigkeiten mit unterschiedlichen CED vorgenommen werden. G. Gee zeigte, dass unter Vernachlässigung der Entropie der elastischen Deformation ein Polymer, in dem nur intermolekulare Dispersionskräfte wirken, am stärksten von einem solchen Lösungsmittel gequollen wird, das die gleiche CED wie das Polymer hat (Gee 1943). Danach sollte eine graphische Darstellung des Quellungsgrades über den Löslichkeitsparametern der Lösungsmittel $\delta_{1}$ eine glatte Kurve mit einem Maximum bei $\delta_{1}=\delta_{2}$ ergeben, wobei $\delta_{2}$ der Löslichkeitsparameter des Polymers ist. Werden die Lösungsmittel allein nach ihren CED ausgewählt, so zeigt sich im Allgemeinen kein derartiger Kurvenverlauf. Um zu aussagekräftigen Ergebnissen zu gelangen, müssen Lösungsmittel benutzt werden, die sich wenig in ihrer chemischen Struktur
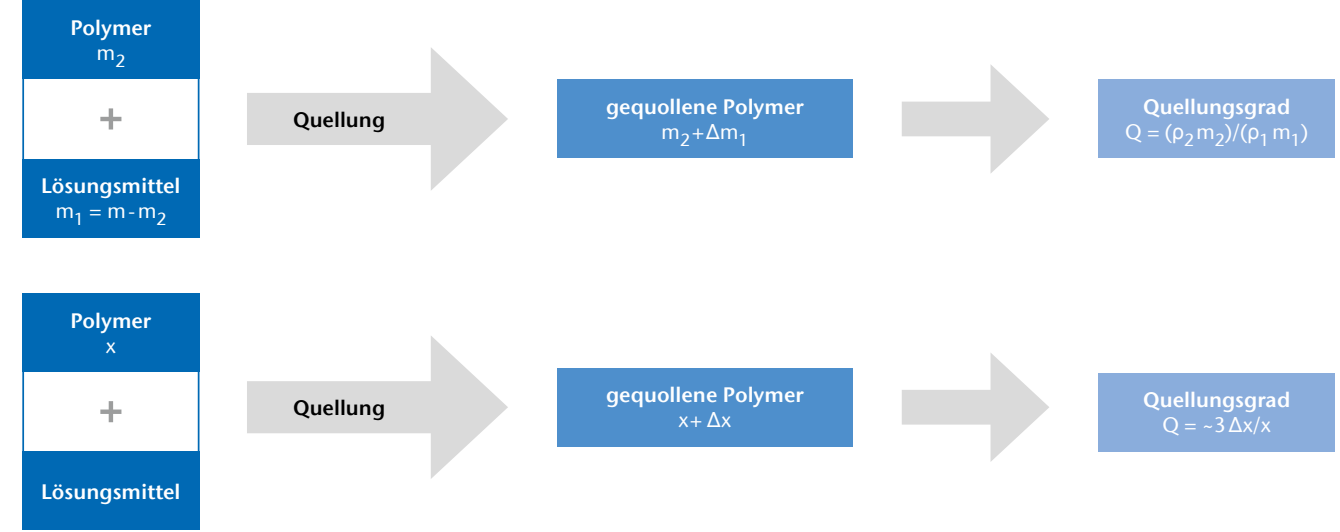
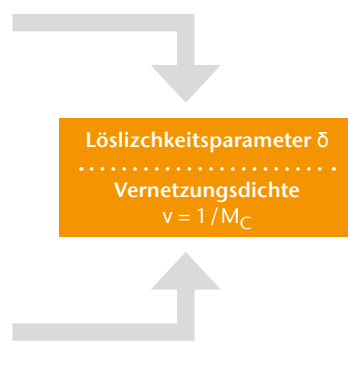
voneinander unterscheiden und eine gewisse Ähnlichkeit mit den Polymersegmenten aufweisen. Durch eine Vielzahl von experimentellen Untersuchungen wurde gezeigt, dass die Löslichkeitsparameter allein nur beschränkte Aussagekraft besitzen. Untersuchungen der Löslichkeitseigenschaften von polaren Polymeren haben begrenzte empirische Korrelationen der Lösungskraft mit der CED, der Gestalt und Größe der Lösungsmittelmoleküle, des Gehalts an Wasserstoffbrückenbindungen im Lösungsmittel, dem Dipolmoment und der Natur der funktionellen Gruppen gezeigt. Mit der Annahme, dass

(4) $\chi_{1 \mathrm{H}}=\chi_{\mathrm{s}}+\frac{\mathrm{V}_{1}}{\mathrm{RT}}\left(\delta_{1}-\delta_{2}\right)^{2}$

ist (hierbei ist $\mathrm{V}_{1}$ das Molvolumen, $\chi_{1 \mathrm{~s}}$ der Entropieterm und $\chi_{1 \mathrm{sH}}$ der Enthalpieterm des Wechselwirkungsparameters $\chi_{1}$ ), zeigten G.M. Bristow und W.F. Watson, dass ein Auftragen der rechten Seite der durch Umformung von Gleichung (4) gebildeten Beziehung

(5) $\frac{\delta_{1}^{2}}{\mathrm{RT}}-\frac{\chi}{\mathrm{V}_{1}}=\frac{2 \delta_{2}}{\mathrm{RT}} \delta_{1}-\frac{\delta_{2}^{2}}{\mathrm{RT}}-\frac{\chi_{\mathrm{S}}}{\mathrm{V}_{1}}$

gegen $\delta_{1}$ eine Gerade ergibt, deren Anstieg und Abschnitt ein Maß für $\delta_{2}$ ist (Bristow und Watsan 1958). Sie nahmen dabei an, dass der Term $\chi / V_{1}$ gegen $\delta_{2}{ }^{2} / R T$ vernachlässigt werden kann. Diese Methode der Darstellung experimenteller Daten sollte es ermöglichen, Lösungsmittel, die durch spezifische Wechselwirkungen eine sehr hohe Quellung hervorrufen (z.B. Chloroform), in demselben Bild darzustellen wie andere relativ schwache Lösungsmittel. Eine kritische Untersuchung dieser Methode enthüllt, dass ihr Erfolg nur scheinbar ist. Die Größe von $\chi$ ist vergleichbar mit der von $\chi_{\mathrm{s}}$. Der Wert von $\chi_{\mathrm{s}}$ kann im Fall von Polymer-Flüssigkeitssystemen, die spezifische Wechselwirkungen aufweisen, den von $\chi$ sogar übersteigen. Das physikalische Problem der Erfassung von spezifischen Wechselwirkungen ist noch nicht befriedigend gelöst, und kein mathematischer Algorithmus bei der Auswertung der Messergebnisse ist geeignet, die Situation zu verbessern. Da die CED von Polymeren nicht direkt aus Verdampfungsexperimenten ermittelt werden kann, wurden CED-Terme für verschiedene molekulare Gruppen aus Dampfdruckmessungen von verdampfbaren Verbindungen übertragen und zur Bestimmung der CED von Polymeren benutzt.

$6 \chi_{1 \mathrm{H}}=\frac{\mathrm{V}_{1}}{\mathrm{RT}}\left[\left(\frac{\Delta \mathrm{E}_{1}}{\mathrm{~V}_{1}}\right)^{1 / 2}-\left(\frac{\Delta \mathrm{E}_{2}}{\mathrm{~V}_{2}}\right)^{1 / 2}\right]^{2}=\frac{\mathrm{V}_{1}}{\mathrm{RT}}\left(\delta_{1}-\delta_{2}\right)^{2}$,

wobei $\Delta \mathrm{E}$ die Verdampfungsenergie und $\mathrm{V}$ das Molvolumen ist. Diese sog. Scatchard-Gleichung ist gleichbedeutend mit der Aussage, dass die Größe $(\Delta \mathrm{E} \mathrm{V})^{1 / 2}$ eine additive Eigenschaft ist (Scatchard 1931). Es lässt sich ein Satz von additiven Konstanten finden, der die Berechnung von $\delta_{2}$ erlaubt. Sie werden als molare Anziehungskonstanten $\mathrm{F}$ bezeichnet. $\delta_{2}$ ist dann gegeben durch

$$
\text { (7) } \delta_{2}=\frac{\sum \mathrm{F}}{\mathrm{V}_{2}} \text {, }
$$

wobei über alle in der betreffenden Substanz enthaltenen Gruppen zu summieren ist. Für eine Reihe von Gruppen sind die molaren Anziehungskonstanten $\mathrm{F}$ bestimmt und mit diesen Werte von $\delta_{2}$ für ausgewählte Polymere berechnet worden. Die Übereinstimmung der berechneten mit den aus Quellungsmessungen beobachteten Werten ist z.T. befriedigend, aber nicht verallgemeinerbar (Scatchard 1931). Für PE wurden keine vergleichbaren Ergebnisse in der Literatur gefunden.

\section{BESTIMMUNG DER VERNETZUNGSDICHTE}

Die Vernetzungsdichte $v_{\mathrm{e}}$ bzw. das Netzkettenmolekulargewicht $M_{c}=1 / v_{e}$ kann durch physikalische oder durch chemische Methoden bestimmt werden. (Der Index e kennzeichnet die effektive, d.h. die mechanisch wirksame Vernetzungsdichte.) Die beiden wichtigsten physikalischen Methoden sind die Messung der Gleichgewichtsquellung und Spannungs-Deformationsmessungen. Aus dem Quellungsgrad einer im Quellungsgleichgewicht befindlichen vernetzten Polymerprobe kann $v_{\mathrm{e}}$ nach der Flory-Rehner-Gleichung ermittelt werden:

$8-\ln \left(1+\frac{1}{Q_{m}}\right)+\frac{1}{1+Q_{m}}+\frac{\chi}{\left(1+Q_{m}\right)^{2}}=\frac{\rho_{2} V_{1}}{M_{c}}\left[\frac{2}{f} \frac{1}{1+Q_{m}}-\frac{1}{\left(1+Q_{m}\right)^{1 / 3}}\right]$

Bei der Anwendung der Beziehung (8) muss der Wechselwirkungsparameter $\chi$ für das betreffende Polymer-Lösungsmittel-System bekannt sein. Die Bestimmung von $\chi$ für PE- und räumlich vernetzte PU-Lösungsmittel-Systeme ist allerdings nicht ohne weiteres möglich, da die bekannten Methoden der $\chi$-Bestimmung, wie die Messung des Dampfdrucks, der Viskosität, des osmotischen Drucks von Polymerlösungen und die Entquellungsmethode nach R.F. Boyer, das unvernetzte Polymerisat benötigen (Boyer 1943). Oft wird sogar gerade andersherum der $\chi$-Parameter von vernetzten Polymer-Flüssigkeitssystemen aus Gleichung (8) berechnet, wobei die Vernetzungsdichte mit einer anderen Methode bestimmt wurde. Der maximale Quellungsgrad $Q_{m}$ ist umso größer, je geringer die Vernetzungsdichte und je kleiner der Wechselwirkungsparameter ist.

Eine Abschätzung der Größenordnung von $\chi$ kann nach Gleichung (4) erfolgen, wenn $\delta_{1}$ und $\delta_{2}$ bekannt sind. Dabei kann für $\chi_{\mathrm{s}}$ nur ein in der Literatur angegebener, für spezielle Systeme abgeschätzter Wert, der als von der CED unabhängig angesehen wird, benutzt werden.

Nach der statistischen Theorie der Kautschukelastizität kann die Vernetzungsdichte aus der an einer vernetzten Polymerprobe angelegten Spannung $\tau$ und der durch diese Spannung bewirkten relativen Deformation $\lambda$ der Probe bestimmt werden. Die Zusammenhänge für einfache 
Dehnung und Kompression werden durch folgende Gleichung zum Ausdruck gebracht:

$$
9 \tau=v_{\mathrm{e}} \mathrm{RT}\left(\lambda-\lambda^{-2}\right) \text {. }
$$

Der Index e soll auch hier andeuten, dass nur Vernetzungsstellen gemessen werden können, die physikalisch effektiv sind. P.J. Flory, A.F. Tobolsky, S. Kästner haben einen sogenannten Front- oder Dilatationsfaktor eingeführt, um die vom frei beweglichen Zustand abweichende Netzkettenkonformation zu berücksichtigen (Flory 1959, Tobolsky 1961, Kästner 1958). Die bei der Ableitung der Gleichung (9) gemachte Voraussetzung, dass zwischen den Netzketten höchstens vernachlässigbar geringe intermolekulare Wechselwirkungen bestehen, ist in vielen Fällen nicht erfüllt. Bei gequollenen Netzwerken wird infolge einer Verminderung der intermolekularen Wechselwirkungen durch das zur Quellung verwendete Lösungsmittel zum Teil eine bessere Übereinstimmung der experimentellen Ergebnisse mit den theoretischen Beziehungen beobachtet. In diesem Fall gilt für Gleichung (9) die Beziehung:

(10) $\tau_{0}=v_{\mathrm{e}} \mathrm{RT} \Phi_{2}^{-1 / 3}\left(\lambda-\lambda^{-2}\right)$,

wobei $\Phi$ der Volumenbruch und $\tau_{0}$ die angewandte Kraft pro Flächeneinheit der ungequollenen, nicht deformierten Probe ist.

\section{ORIENTIERENDE MESSUNGEN AN POLYURETHANEN}

In der Literatur ist eine Reihe von Untersuchungen zum Quellverhalten von PU-Systemen beschrieben. (Z.B. Makitra 2005, Pacheco 2009). Zur Überprüfung der Anwendbarkeit der oben beschriebenen Methoden auf reale Systeme wurde das Quellverhalten von Modell-PU in einer Vielzahl von niedermolekularen Substanzen untersucht. Es zeigt sich, dass der Wert der CED allein nicht ausreicht, um Vorhersagen über das Löslichkeits- bzw. Quellverhalten des Polymers machen zu können.
Die Methode der Bestimmung von $v_{\mathrm{e}}$ aus Quellungsdaten nach Gleichung (8) kann für die untersuchten ModellPU nicht angewendet werden, da diese sich, wie Messungen des Torsionsmoduls in Abhängigkeit von der Temperatur zeigen, bei RT (Messtemperatur) nicht im inneren Gleichgewicht befinden. Aus gleichem Grund lässt sich auch aus dem Spannungs-Dehnungsverhalten dieser PU kein sinnvoller Wert für $v_{\mathrm{e}}$ ableiten.

Von den bisher bekannten Verfahren der Bestimmung der CED von Polymeren erscheint die Methode nach $G$. Gee die am besten geeignete. Hierzu wer-
Die Dichte- und $\delta_{1}$-Werte der benutzten Lösungsmittel wurden der Literatur entnommenen (Bristow und Watson 1958). Die Gee'sche Behandlung für die Ermittlung der CED von Polymeren verlangt aber, dass die allgemeine Natur der Polymer-FlüssigkeitsWechselwirkung in allen Systemen möglichst ähnlich ist. Deshalb wurde eine vergleichende Untersuchung des Quellverhaltens in einer Reihe von Estern durchgeführt. Die Eigenschaftswerte der Ester sind Literaturdaten (Hansen 2012).

Die Bestimmung des maximalen Quellungsgrades erfolgte nach der

\begin{tabular}{|c|c|c|}
\hline Probe & Zusammensetzung & $\delta_{2} /\left(\mathrm{cal} \cdot \mathrm{cm}^{-3}\right)^{1 / 2}$ \\
\hline $\mathrm{PU}_{1}$ & $\begin{array}{l}63 \mathrm{~g} \text { Polybutylenglycol } \\
22.5 \mathrm{~g} \text { Desmodur }^{\circledast} 44 \\
5.5 \mathrm{~g}^{\text {Butandiol } 1.4}\end{array}$ & 9.4 \\
\hline $\mathrm{PU}_{2}$ & $\begin{array}{l}63 \mathrm{~g} \text { Polybutylenglycol } \\
39.6 \mathrm{~g} \text { Desmodur }{ }^{\circledast} 44 \\
10 \mathrm{~g} \text { Butandiol } 1.3\end{array}$ & 9.2 \\
\hline$S$ & $\begin{array}{l}102 \mathrm{~g} \text { Glyzerin-Propylenoxid-Polyether } \\
57 \mathrm{~g} \text { Desmodur }{ }^{\oplus} 44 \\
6.1 \mathrm{~g} \text { Triethanolamin }\end{array}$ & 9.0 \\
\hline
\end{tabular}

Tab. 1) Zusammensetzung der Modell-Polymere und ermittelte Löslichkeitsparameter $\delta_{2}$.

den die Gleichgewichtsquellungswerte $\mathrm{Q}_{\mathrm{m}}$ in einer Reihe von Lösungsmitteln mit unterschiedlicher CED ermittelt. Die Zusammensetzung der drei an der TH Wildau hergestellten Modellproben $\mathrm{PU}_{1}, \mathrm{PU}_{2}$ (Elastomer) und $\mathrm{S}$ (Schaum) ist der Tabelle 1 zu entnehmen: gravimetrischen und der mikrooptischen Methode. Die Durchführung der Gleichgewichtsquellungsmessung nach der gravimetrischen Methode ist relativ einfach und dennoch recht genau. Die größte relative Abweichung zwischen zwei gleichen Proben be-

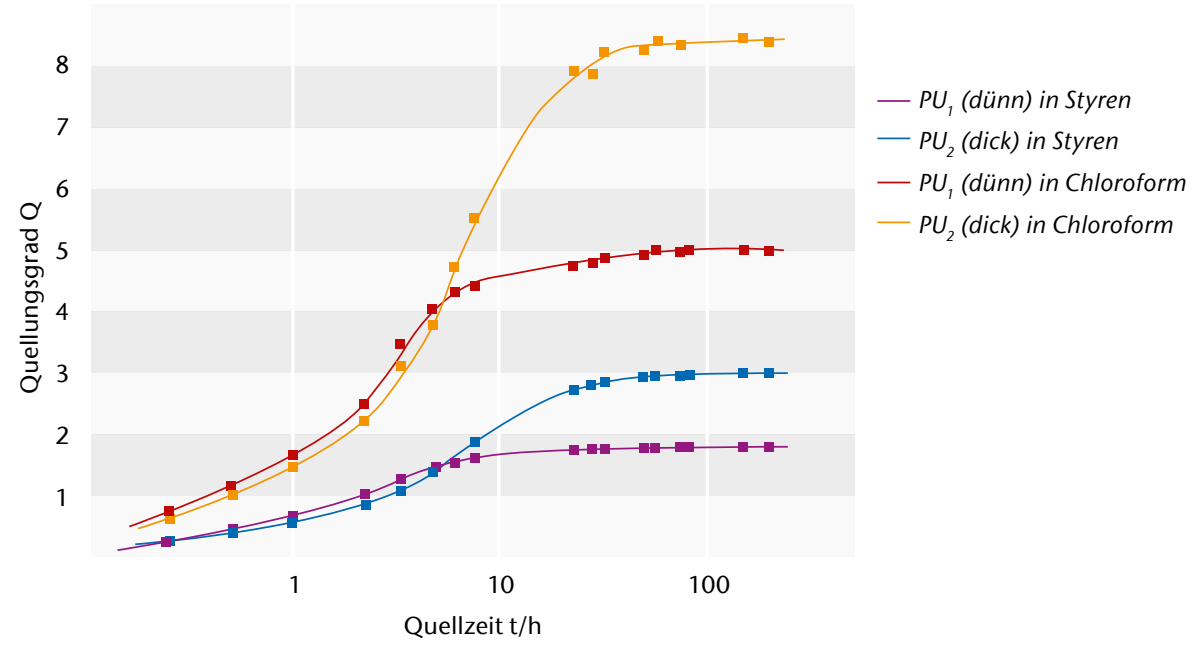

$A b b$. 2) Abhängigkeit des Quellungsgrades $Q$ von der Quellzeit $t$. 
trug ca. $4 \%$. Hauptsächlich wird sie durch das nicht exakt reproduzierbare Abtrocknen der Probenoberfläche nach der Gleichgewichtsquellung verursacht.

UHMPE-Proben während der Quellung herausgelöst, und selbst die bestrahlten UHMWPE-Proben erleiden während der Quellung eine nicht zu vernachlässigende Abnahme ihrer Masse.

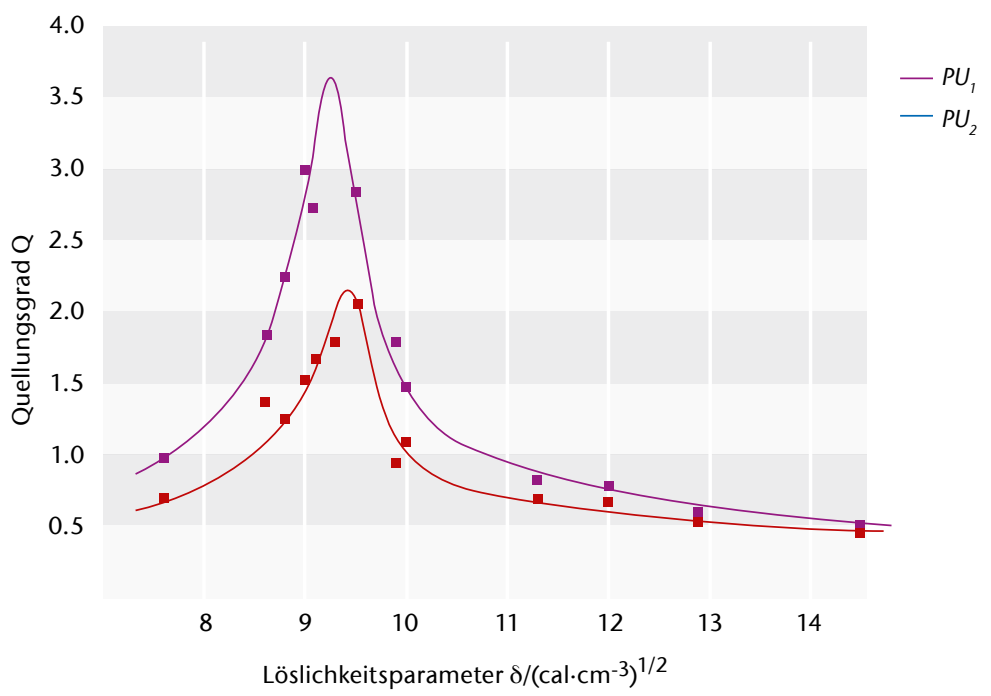

$A b b .3)$ Quellungsgrad der $P U_{1}$ und $P U_{2}$ in Abhängigkeit vom Löslichkeitsparameter $\delta_{1}$ nach Bristow und Watson (1958).

Um zu prüfen, wie schnell das Quellungsgleichgewicht erreicht wird, sind vollständige Quellungs-Zeit-Kurven aufgenommen worden. Beispiele sind in Abb. 2 dargestellt. Die zylindrischen Proben hatten eine Höhe von $2.5 \mathrm{~mm}$ und einen Durchmesser von $15 \mathrm{~mm}$ für $\mathrm{PU}_{1}$ und eine Höhe von $4 \mathrm{~mm}$ bei gleichem Durchmesser für $\mathrm{PU}_{2}$. Wie Abb. 2 zeigt, wird der Gleichgewichtsquellungswert nach etwa 2 bis 3 Tagen erreicht. Bei sämtlichen Messungen des maximalen Quellungsgrades wurden die Proben mindestens $5 \mathrm{~d}$ mit dem Lösungsmittel in Kontakt gelassen und die Probenabmessungen stets kleiner gehalten als die oben angegebenen.

Im Anschluss an die Quellungsmessung wurde durch Desorption geprüft, ob durch das Lösungsmittel Bestandteile der Polymerprobe herausgelöst wurden. Die hier untersuchten Proben erreichten nach hinreichend langer Zeit (ca. 1 Woche) im Rahmen der Messgenauigkeit wieder ihre Ausgangsmasse. Sämtliche Messungen sind bei $23{ }^{\circ} \mathrm{C}\left( \pm 2{ }^{\circ} \mathrm{C}\right)$ durchgeführt worden. Wie sich zeigen wird, sind die Verhältnisse beim UHMWPE selbst nach einer gewissen Strahlenvernetzung weit komplizierter. Ein relativ großer Masseanteil wird aus den
In Abb. 3 sind die Quellungsgrade Q von $\mathrm{PU}_{1}$ und $\mathrm{PU}_{2}$ über den $\delta_{1}$ der jeweiligen Lösungsmittel aufgetragen. Halogenierte Kohlenwasserstoffe zeigen im Allgemeinen ein besonders starkes Quellungsvermögen, während z.B. zyklische Verbindungen eine rela-
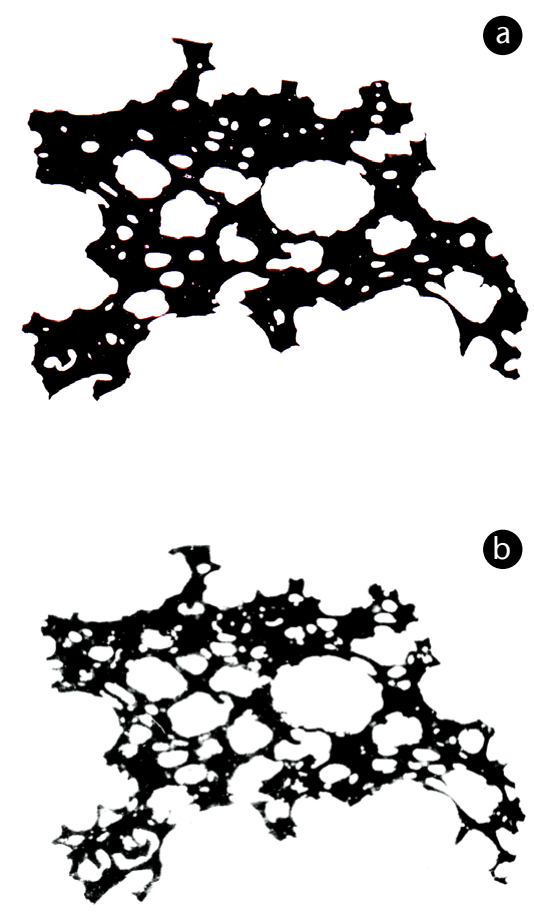

Abb. 4) PU-Schaumteilchen S, a) vor und b) nach 15 min Quellung in Amylformiat tiv schwache Quellung hervorrufen. Diese Lösungsmittel und andere, die eine spezifische Wechselwirkung aufweisen und nicht in das allgemeine Schema des Quellverhaltens passen (z.B. Ameisensäure wegen Umesterung), wurden in Abb. 3 nicht eingetragen. Für die Quellungsmessung an Schaumstoffen eignet sich die gravimetrische Methode nicht.

Hier wird besser eine mikrooptische Messmethode angewendet. Als Messproben werden Schaumteilchen von etwa 1 bis $2 \mathrm{~mm}$ Durchmesser und 0.1 bis $0.2 \mathrm{~mm}$ Dicke verwendet, die aus Mikroschnitten gewonnen werden. Zur Bestimmung des Quellungsgrades wurden die Proben einmal vor und einmal 15 min nach dem Einfüllen des Lösungsmittels mit Hilfe eines Mikroskops abgebildet (Abb. 4). Aus der linearen relativen Dimensionsänderung der Probe $\Delta x / x$ wird nach Gleichung (3) der Quellungsgrad berechnet. Zur Überprüfung der Isotropie der Quellung sind die Längenänderungen der Probe $S$ in fünf verschiedenen Richtungen gemessen worden. Die Unterschiede zwischen diesen einzelnen Messungen lagen innerhalb der Messgenauigkeit. Bei der Untersuchung von sehr kleinen Probekörpern im Bereich von ca. 50 bis $100 \mu \mathrm{m}$ zeigte sich eine gewisse Anisotropie der Längenausdehnung im Lösungsmittel. Gemäß der Abb. 3 analogen Auswertung ergibt sich für den PU-Schaum $\delta_{s}=9.0$ $\pm 0.1\left(\mathrm{cal} \cdot \mathrm{cm}^{-3}\right)^{1 / 2}$.

Zum Vergleich der Messmethoden wurde die Quellung von $\mathrm{PU}_{2}$ in Estern auch mit der mikrooptischen Methode bestimmt (Abb. 5). Es wurden ca. $30 \mu \mathrm{m}$ dicke Proben verwendet. Die Quellungsgrade, die nach der volumetrischen Methode bestimmt wurden, sind erwartungsgemäß stets niedriger als die massenmäßig ermittelten. Die relativen Verhältnisse der Abhängigkeit der Quellungsgrade von den Löslichkeitsparametern der Quellungsmittel bleiben jedoch die gleichen, sodass beide Methoden die gleiche CED für das Polymer liefern.

Allerdings kann man sich auch vorstellen, dass eine gewisse Menge an niedermolekularer Flüssigkeit vom Polymer aufgenommen werden kann, 


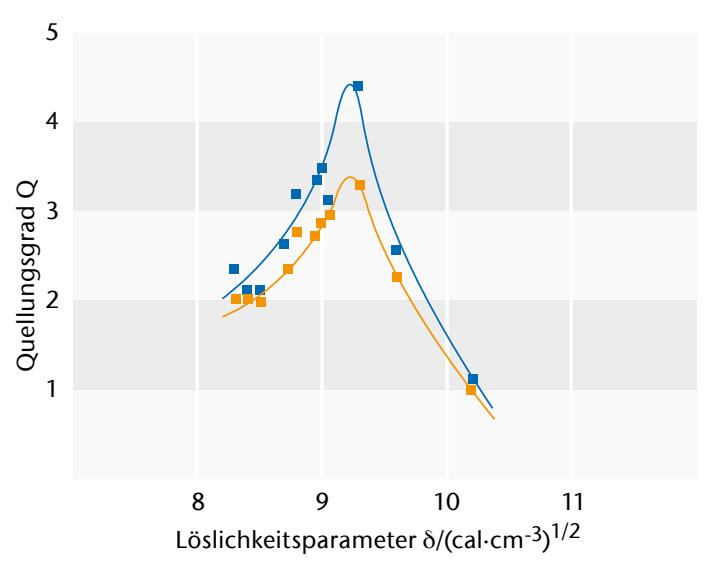

Abb. 5) Abhängigkeit des Quellungsgrades Q von PU $E_{2}$ von den Löslichkeitsparametern der Ester. Vergleich von gravimetrischer und mikrooptischer $\square$ Messmethode. ohne dass sich dabei das Volumen der makromolekularen Komponente verändert, d.h., dass erst gewisse "Hohlräume" in der Polymermatrix ausgefüllt werden. Bei der quantitativen Bestimmung der Quellung muss also stets geprüft werden, ob die Annahme der Additivität der Volumina zu Recht gemacht wurde. Wie erwartet unterscheiden sich die CED der einzel-
CED allein weniger geeignet. Dass von den untersuchten PU das Elastomer $\mathrm{PU}_{1}$ den größten CED-Wert besitzt, kann auf Grund der Zusammensetzung der drei PU-Systeme erwartet werden. So sind beim Elastomer $\mathrm{PU}_{1}$ im Gegensatz zu den beiden anderen PU alle drei Ausgangskomponenten lineare Produkte. Seitengruppen, wie die $\mathrm{CH}_{3}$-Gruppen des Butandiols-1.3 beim Elastomer $\mathrm{PU}_{2}$
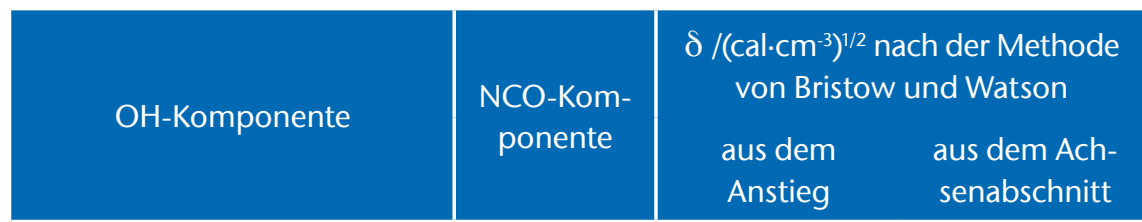

Diethylenglykol

Triethylenglykol

Tetraethylenglykol

Hexaethylenglykol

Octaethylenglykol

Decaethylenglykol

Polyoxytetramethylenglykol

Polyoxypropylenglykol

Polydiethylenadipinat
9.2

9.7

10.4

10.7

Triphenyl-
methan-
$4,4^{\prime}, 4^{\prime \prime}{ }^{\prime \prime}$-trii-
socyanat

10.5

10.8

10.5

10.6

10.8

10.8

10.9

10.9

\section{5}

\section{Toluy-}

len-2,4-

diisocyanat

\section{8}

12.0
Die Anwendung der Flory-RehnerGleichung (8) und der SpannungsDeformationsbeziehung (10) zur Bestimmung der Vernetzungsdichte verlangt, dass sich das Polymer bei Messtemperatur im inneren Gleichgewicht befindet. Wie entsprechende Messungen zeigten, befinden sich die vorliegenden PU-Systeme bei Raumtemperatur nicht im inneren Gleichgewicht.

\section{V.II UNTERSUCHUNGEN AN POLYETHYLEN}

PE, speziell HMWPE und UHMWPE, sind sowohl bei der Bestimmung der Kohäsionsenergiedichte als auch bei der Ermittlung der Vernetzungsdichte noch schwerer zugänglich, obwohl im Gegensatz zum PU die Ketten einheitlich aus kleinen unpolaren Monomerbausteinen bestehen. Auf Grund der Regelmäßigkeit und Symmetrie der Anordnung der monomeren Bausteine $\left(\mathrm{CH}_{2}\right.$-Gruppen) in der Kette, d.h. der Zick-Zack-Konformation der Ketten sowie der Drehbarkeit der Kettenglieder um die Einfachbindungen (-CC-), sind diese aber in der Lage, trotz der geringen Energie der einzelnen zwischenmolekularen Wechselwirkungen $(\sim 2 \mathrm{~kJ} / \mathrm{mol})$ bei Raumtemperatur, geordnete bzw. kristalline Bereiche in Form von Lamellen zu bilden. Der Schmelzbereich der Kristallite liegt je nach Kettenlänge, einschließlich Verzweigungen, und Kristallisationsbedingungen zwischen ca. $110{ }^{\circ} \mathrm{C}$ und $140{ }^{\circ} \mathrm{C}$, wobei UHMWPE die höchste Schmelztemperatur aufweist. Die Löslichkeit bzw. Quellbarkeit von PE in organischen Lösungsmitteln ist deshalb bei Raumtemperatur äußerst gering. Da das "Eindringen“ von niedermolekularen Substanzen in das Polymergefüge vor allem durch die Wechselwirkung zwischen Lösungsmittel und Polymer bestimmt wird und bevorzugt in den amorphen Bereichen erfolgt, gelingt das Lösen von PE nur in wenigen siedenen Lösungsmitteln wie Xylol, Decahydronaphthalen (Decalin) oder Trichlorbenzol. verhindern auf Grund sterischer Effekte, dass die zwischenmolekularen Kräfte über die ganze Länge der Kettenmoleküle voll zur Wirkung kommen, und verringern so den Wert der CED des Polymeren. Zum Vergleich sind in Tabelle 2 einige Literaturwerte für die Löslichkeitsparameter von PU zusammengestellt.
Orientierende Untersuchungen zum Quellverhalten von HMWPE und UHMWPE wurden in siedendem Xylol an folgenden Proben vorgenommen: unbestrahltes Lupolen L1, bestrahltes 
Lupolen L2, unbestrahltes GUR GT1, bestrahltes GUR GT2 und GT3. Die Modellproben wurden von Frau Dr. von der Ehe, Fachbereich 3.4, Sicherheit von Lagerbehältern, BAM, vorbereitet und die Vernetzungsgradbestimmung durchgeführt (Wolff 2013). Dazu wurden die Proben nach der Bestimmung der Ausgangsmasse $m_{A}$ in einem kleinen Maschendrahtkäfig in einen Rundkolben mit Rücklaufkühler wie 2,2'-Methylen-bis-(4-methyl-6 tert.butylphenol) bzw. 3-(3,5 di-tert. butyl-4-hydroxiphenyl)-propionat erfordert. In den durchgeführten Vorversuchen wurden derartige Stabilisatoren nicht hinzugefügt. Der Abb. 6 ist zu entnehmen, dass die Strahlenbelastung (gemäß der Probendaten) zu einer ernormen Zunahme des unlöslichen Anteils führt, d.h. es erfolgt bei der Bestrahlung eine hauptvalenzmä-

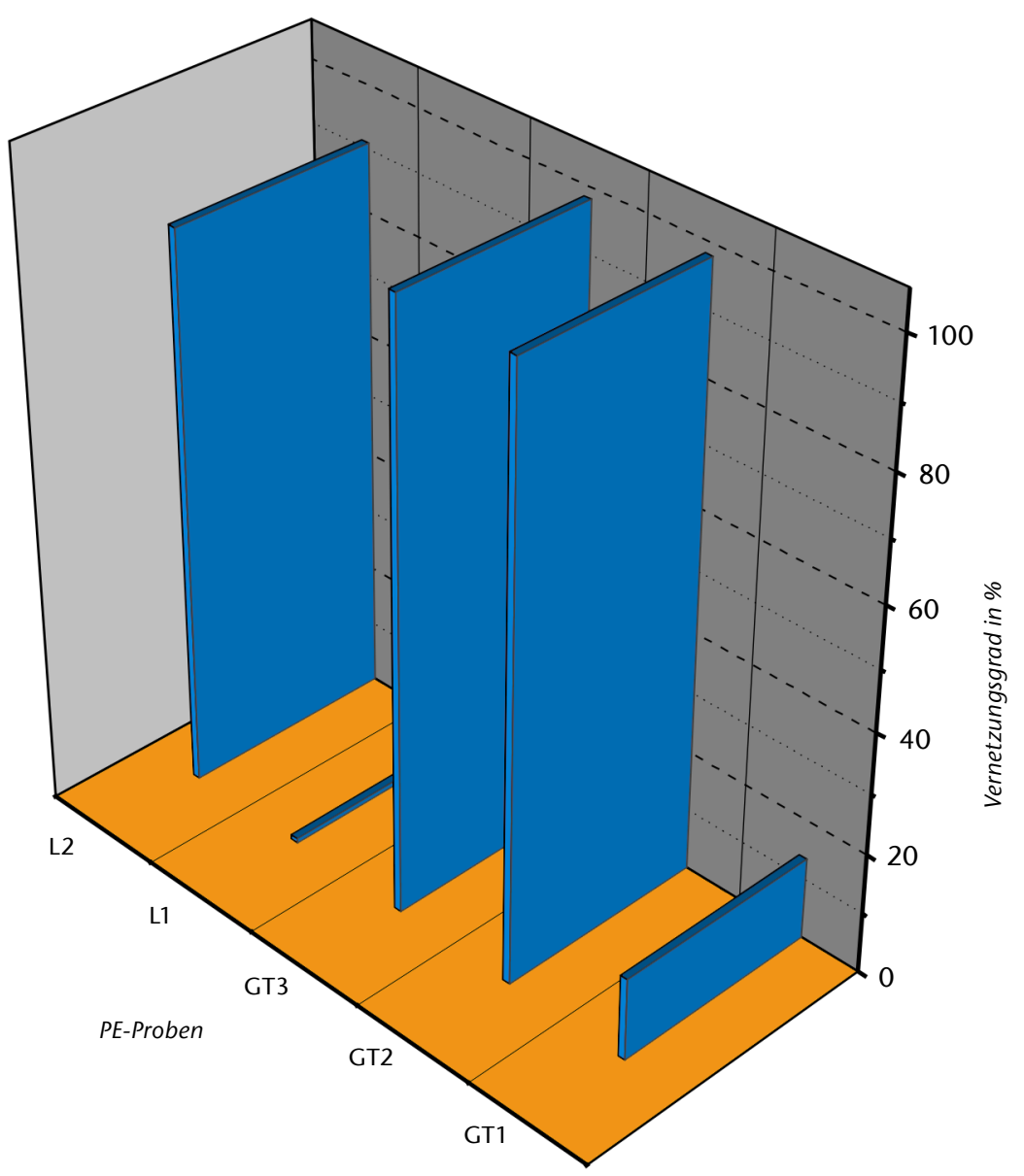

Abb. 6) Vernetzungsgrad G in Anlehnung an DIN 16892 für GUR- und Lupolen-Proben.(Wolff 2013).

in das kochende Xylol gebracht und nach $8 \mathrm{~h}$ die Restmasse des jeweiligen Probekörpers bestimmt. In Anlehnung an die DIN 16892:2000-07 wird ein Maß für den Vernetzungsgrad $G$ des UHMWPE aus dem im siedenden Xylol unlöslichen Masseanteil $m_{u}$ nach: $G=$ $\mathrm{m}_{\mathrm{U}} / \mathrm{m}_{\mathrm{A}} \cdot 100$ bestimmt.

Die für die einzelnen Proben bestimmten Werte sind in Abb. 6 als Balkendiagramm dargestellt. Erwähnt werden muss, dass nach DIN 16892 die Lagerung in siedendem Xylol die Zugabe von $1 \%$ Antioxidationsmittel ßige Verknüpfung fast aller Kettenmoleküle untereinander, wobei der Vernetzungsgrad (unlöslicher Anteil) des GUR (98\%) wiederum größer ist als beim Lupolen (91\%). Eine quantitative Analyse kann nur im Zusammenhang mit Messergebnissen anderer Strukturmethoden gegeben werden, einschließlich der Unterscheidung von strahlenchemischer Vernetzung und Kettenspaltung. Ein analytisches Maß für den jeweiligen Anteil lässt sich aus dieser Art von Quellungsmessungen allein nicht ableiten. Die wie oben beschriebene Bestimmung des un-
Iöslichen Masseanteils berücksichtigt nicht die durch die Strahlenbelastung erfolgte Kettenspaltung und damit eine Erhöhung der löslichen Anteile. So kann der Vernetzungsgrad $G$ der beiden strahlenvernetzten Proben aus Lupolen und GUR nicht unmittelbar miteinander verglichen werden. Eine Korrektur durch das Herauslösen der Kettenspaltungsprodukte ist jedoch vor allem beim Lupolen wegen der äußerst geringen Masse der unlöslichen Bestandteile nicht sinnvoll. Weiterhelfen könnte eine quantitative Analyse der Molmasse und Molmassenverteilung des Iöslichen Anteils, die allerdings spezieller vorbereitender Arbeiten bedarf.

Umgangen werden einige der Probleme durch die Bestimmung der Vernetzungsdichte (Vernetzungsgrad) mit Hilfe der mikrooptischen Ausdehnungsmessung der gequollenen Proben nach Gleichung (3). Dazu wurden Scheiben von ca. $4 \mathrm{~mm}$ Durchmesser ausgestanzt. Der Durchmesser der einzelnen Proben wird vor der Quellung mit Hilfe eines Mikroskops genau ausgemessen. Die Kontur wird mit einer kreisförmigen Simulation festge-

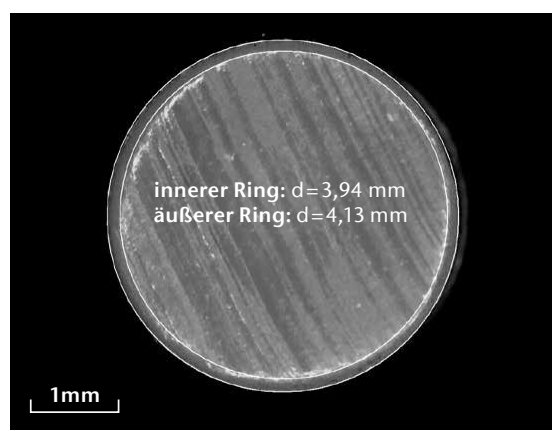

Abb. 7) PU-Schaumteilchen S, vor und nach $15 \mathrm{~min}$ Quellung in Amylformiat

legt. Ein entsprechendes Rechenprogramm ermöglicht die Bestimmung der markierten Fläche (einschließlich des Durchmessers). Der Quellungsgrad Q kann dann aus dem Flächenmaß der Probe vor und nach der Quellung ermittelt werden. Hierbei sollte ein Herauslösen von kürzeren Ketten oder Kettenteilen weniger Einfluss auf das Ergebnis haben.

Beobachtet wurde mit einem WildMikroskop durch ein Objektiv $12 \times$ mit Hilfe einer dhs Microcam 1.3. In Abb. 7 ist ein Beispiel für eine Probe vor 
der Quellung zu sehen. Die entsprechenden Messungen der gequollenen Proben bedürfen noch einer Reihe von vorbereitenden Untersuchungen. Dabei spielt die zeitliche Stabilität des Quellungsgleichgewichts und ihre Abhängigkeit von der Temperatur (vor allem mit Erreichen der Kristallisationstemperatur) eine entscheidende Rolle. Derartige Messungen wurden begonnen. Für die Deutung der mit dieser Methode zu erwartenden Ergebnisse gilt jedoch prinzipiell das oben zu der gravimetrischen Messung des Vernetzungsgrades bereits Dargelegte.

\section{V.III. FAZIT}

Die CED ist geeignet, das Verhalten von PU gegenüber verschiedenen Lösungsmitteln vorherzusagen. Der experimentelle Zugang erfolgt über Quellungsmessungen in Lösungsmitteln mit unterschiedlichem $\delta$. Es wird die gravimetrische mit einer mikrooptischen Methode, bei der die Längenausdehnung einer Probe durch die Aufnahme von niedermolekularer Flüssigkeit gemessen wird, verglichen. Die angewendete mikrooptische Quellungsmessung bietet den Vorteil einer genaueren Bestimmung des Quellungsgrades und darüber hinaus die Möglichkeit der Messung an sehr kleinen Probekörpern. So können wegen der diffusionskontrollierten Einstellung des Quellungsgleichgewichts sehr kurze Messzeiten erzielt werden. Die gravimetrische Methode erfordert wegen der größeren Probekörper weit längere Messzeiten. Für die untersuchten Modell-Polyurethane wird ein Wert für $\delta$ von ca. $9\left(\mathrm{cal} \cdot \mathrm{cm}^{-3}\right)^{1 / 2}$ ermittelt. Der Vergleich mit Daten aus der Literatur belegt die Abhängigkeit von der molekularen Struktur der PU. Da sich die untersuchten physikalisch oder chemisch vernetzten PU-Systeme nicht im thermodynamischen Gleichgewicht befinden, lassen sich die Flory-Rehner-Gleichung und entsprechende Spannungs-Deformationsbeziehungen zur Bestimmung von $v$ nicht anwenden.
Da sich PE nur in siedendem Xylol quellen oder lösen lässt, können nur vergleichende Untersuchungen zum Vernetzungsgrad in Anlehnung an DIN 16892 durchgeführt werden. Ausgewertet wird gravimetrisch, da die PE-Proben bei der Quellung ihre Form verlieren. Darüber hinaus werden z.T. erhebliche Anteile von Makromolekülen aus den Messproben extrahiert. Die klassische Theorie des Quellverhaltens von Polymeren lässt sich nicht anwenden, da HMWPE und UHMWPE lineare Systeme sind und nur durch Verschlaufungen physikalische Netzwerke bilden. Der relative Einfuß von Gamma-Bestrahlung auf die Vernetzungsstruktur von PE konnte durch die Bestimmung des Vernetzungsgrades eindruckvoll belegt werden. Die quantitative Bestimmung der Vernetzungsdichte von PE-Systemen bedarf auf Grund ihrer komplizierten Morphologie besonderer Anstrengungen. Entsprechende Arbeiten wurden bereits begonnen.

\section{DANKSAGUNC}

Die Autoren bedanken sich bei Frau Dipl. Ing. Edelgard Lorenz, Fachbereich 6.5, Polymere in Life Science und Nanotechnologie, BAM, für die mikroskopischen Untersuchungen.

\section{LITERATUR}

Apuchtina, N.P, Ehrenburg E.G., Rappoport L.J. (1966): Hochmol. Verb. 8, 1057

Boyer, R.F. (1943): J. Chem. Phys. 13, 363

Bristow, G.M., Watson, W.F. (1958): Trans. Faraday Soc. 54,1731

Flory, P.J. (1953): Principles of Polymer Chemistry, New York

Flory, P.J., Hoeve, C.A.J., Ciferri, A. (1959): J. Polymer Sci. 34,337

Forrest, M.J. (2001): Rubber Analysis, Rapra Review Reports 139 , Vol. 12 , No 7,56

Gee, G. (1943): Trans. Inst. Rubber Ind. 18, 266

Hansen, C.M. (2012): Hansen Solubility Parameters: A User's Handbook, Second Edition

Katz, D., Salee, G. (1968): J. Polymer Sci. (A-2) 6, 801

Kästner, S. (1958): Colloid \& Polymer Sci., 157, 133

Makitra, R.G., Pristanskii, R.E., Evchuk, I.Yu. (2005): Polymer Sci. 47, No 11, 1987

Pacheco, M.F.M., Bianchi, O., Fiorio, R., Zattera, A.J. (2009): J. of Elast. and Plas, 41

Scatchard, G. (1931): Chem. Reviews 8, 321

Tobolsky, A.F., Carlson, D.W., Indictor, N. (1961): J. Polymer Sci. 54, 175

Wolff, D., Von der Ehe, K., Jaunich, M., Böhning, M., Goering, H. (2013): I. of ASTM Internat., Vol. 25, STP 1547, 211

\section{AUTOREN}

Dipl.-Ing. Vesela Stoycheva

Fachbereich Ingenieur- und Naturwissenschaften Technische Hochschule Wildau [FH]

T +49 3375 508-592

vesela.stoycheva@th-wildau.de

Dr. rer. nat. Harald Goering

Fachbereich 3.4, Sicherheit von Lagerbehältern Bundesanstalt für Materialforschung und - prüfung Unter den Eichen 87 12205 Berlin

\section{Dr. rer. nat. Dietmar Wolff}

Fachbereich 3.4, Sicherheit von Lagerbehältern Bundesanstalt für Materialforschung und - prüfung Unter den Eichen 87

12205 Berlin

$\mathrm{T}+4930$ 8104-1341

dietmar.wolff@bam.de

Prof. Dr. rer. nat. Michael Herzog

Fachbereich Ingenieur- und Naturwissenschaften

Technische Hochschule Wildau [FH]

$\mathrm{T}+493375$ 508-332

michael.herzog@th-wildau.de 